\title{
Isoliquiritigenin attenuates LPS-induced acute kidney injury through suppression of HMGB1 pathway in renal tubular against ferritinophagy
}

\section{Yun Tang}

University of electronic science and technology of China

\section{Yanmei Wang}

Affiliated hospital of north sichuan medical college

Chan Wang

University of electronic science and technology of China

\section{Meidie Yu}

University of eletronic science and technology of China

Li Li

Sichuan University

\section{Sipei Chen}

University of electronic science and technology of China

\section{Guisen Li}

Sichuan Academy of Medical Sciences and Sichuan People's Hospital

\section{Li Wang}

Sichuan Academy of Medical Sciences and Sichuan People's Hospital

\section{Mengyi Xie}

Affiliated hospital of north sichuan medical college

Yi Li ( $\square$ liyisn@med.uestc.edu.cn )

Sichuan Academy of Medical Sciences and Sichuan People's Hospital

\section{Research article}

Keywords: Acute kidney injury, Isoliquiritigenin, ferroptosis, ferritinophagy, Autophagy, HMGB1

Posted Date: February 21st, 2020

DOI: https://doi.org/10.21203/rs.2.24196/v1

License: (c) (1) This work is licensed under a Creative Commons Attribution 4.0 International License.

Read Full License 


\section{Abstract}

Septic acute kidney injury (AKI) mainly results in life-threatening renal dysfunction involving renal tubular injury to bring heavy burden to patients in intensive care unit (ICU). However, there is still a lack of therapy to prevent septic AKI effectively and inexpensive. To observe the role and novel mechanism of isoliquiritigenin (ISL) which isolated from the roots of licorice in septic AKI, we used LPS to induce renal tubular injury upon septic AKI both in vitro and in vivo. $50 \mathrm{mg} / \mathrm{kg}$ ISL and $5 \mathrm{mg} / \mathrm{kg}$ Ferrostatin- 1 were once given to the male C57BL/ 6 mice one hour before $1 \mathrm{mg} / \mathrm{kg}$ LPS i.p injection. $50 \mu \mathrm{M}$ and $100 \mu \mathrm{M}$ ISL respectively pre-treat the human renal tubular cells $5 \mathrm{hrs}$ before $2 \mu \mathrm{g} / \mathrm{ml}$ LPS stimulation. We found ISL pretreatment apparently reversed LPS-induced renal dysfunction and ameliorated murine renal tubular injury by suppression HMGB1 pathway. Furthermore, we observed that LPS induced autophagy and ferroptosis in renal tubular, whereas ISL pretreatment significantly suppress autophagy and ferroptosis of renal tubular both in vitro and in vivo. Mechanically, autophagy activated ferroptosis via NCOA4-mediated ferritinophagy. Moreover, HMGB1 is required for ferritinophagy in renal tubular. ISL treatment inhibited the expression of HMGB1. Taken together, these results suggest that ISL protects LPS-induced acute kidney injury through suppression of HMGB1 pathway in renal tubular against ferritinophagy.

\section{Background}

$\mathrm{AKI}$, a major consequence of sepsis, mainly results in increased plasma creatinine. Overall in-hospital mortality rate was $50-60 \%$ and was higher in those with sepsis $(70-80 \%)^{1}$. Patients need to dialysis and transfer to intensive care unit (ICU) so that lead to sanitary and economic problem². As for medium and long-term consequences, the possibility of favours progression to chronic kidney disease (CKD), cardiovascular morbimortality and permanent dialysis dependence will be increased ${ }^{2}$.

Additionally, AKI is an acute inflammatory disease of renal tubular cells through multiple mechanisms, including necrosis (regulated necrosis, RN) and apoptosis². Therefore, Martin-Sanchez, D. et al. pointed that ferroptosis plays the main role in Nephrotoxic Folic Acid-Induced AKI ${ }^{3}$. J. P. Friedmann Angeli, M et.al. found the activation of ferroptosis leads to acute renal injury in mice ${ }^{4}$. We considered highly that ferroptosis involved in AKI.

Ferroptosis, a form of iron-dependent cell death, is identified by accumulated lipid peroxidation and iron ${ }^{5}$. Autophagy is a conversed degradation pathway that maintains cellular homeostasis ${ }^{6}$. ferritinophagy defied as an autophagic phenomenon and degraded via autophagy to release iron ${ }^{7,8}$. In current study, nuclear receptor coactivator 4 (NCOA4) is the cargo receptor for ferritinophagy enriched in autophagosomes ${ }^{7}$. So, we are interested whether ferroptosis and ferritinophagy have a relationship.

High mobility group box 1(HMGB1), as a proinflammatory mediator, releases by damaged cells in inflammatory diseases ${ }^{9}$. Importantly, HMGB1 plays a significant role in sepsis-associated acute kidney injury ${ }^{10}$. As for mechanism, previous works demonstrated that HMGB1 involves in autophagy through 
various pathways ${ }^{11-13}$. But there is a gap between HMGB1 and iron-related cell death. Hence, we considered the potential mechanism that HMGB1 involved in autophagy, ferritinophagy and ferroptosis in LPS-induced AKI.

Isoliquiritigenin (ISL) is one of the bioactive components isolated from the roots of a Chinese traditional herb called licorice ${ }^{14}$. ISL exerted useful biological activities such as anti-inflammatory, anti-oxidative, anti-nephritic and anti-cancer activities ${ }^{15}$. Meanwhile, ISL treatment decreased nitric oxide (NO) production and inhibit autophagy process ${ }^{14,16}$. Moreover, ISL inhibited amount of reactive oxygen species (ROS) production ${ }^{17}$.

In this study, we have now explored the role of autophagy, ferritinophagy and ferroptosis in AKI induced by LPS. Mechanically, autophagy activated ferroptosis via NCOA4-mediated ferritinophagy in septic AKI.

\section{Methods}

Chemicals and reagents

Isoliquiritigenin was obtained from MedChemExpress (10208, MCE, USA). Escherichia coli 0111:B4 LPS was obtained from Sigma-Aldrich (L4130, Sigma, USA).

Animal Model

All procedures were conducted in accordance with National Institutes of Health guidelines for animal care and use, Male C57BL/6 mice (six to eight-week-old, 22-25 g) from the Experimental Animal Center of Sichuan Provincial People's Hospital were treated with a standard laboratory diet. LPS was dissolved in normal saline and ISL was dissolved into $0.5 \%$ Tween-20/saline. AKI mice were given i.p. with LPS. All the mice were randomly divided into six groups $(n=8)$ : control, ISL, Fer, LPS, LPS plus ISL and LPS plus Fer group. LPS was i.p. injected at a dosage of $10 \mathrm{mg} / \mathrm{kg}$. For ISL treatment, $50 \mathrm{mg} / \mathrm{kg}$ ISL was given to the mice via gavage before LPS injection. Fer (Ferrostatin-1, SML0583, Sigma-Aldrich, USA) dosed intraperitoneally with $5 \mathrm{mg} / \mathrm{kg}$ to mice. Mice were sacrificed by cervical dislocation at $8 \mathrm{hrs}$ after LPS injection. At the same time, the kidney and serum samples were collected. The volume of blood samples was limited, thus the samples were randomly chosen from each group for CREA, BUN, SOD, MDA and NO tests.

\section{Cell Cultures And Treatments}

The human kidney epithelial tubular cell line HK2 (ATCC® CRL-2190 ${ }^{\mathrm{TM}}$ ) was grown in DME/F-12 (SH30023.01, HyClone, USA), supplemented with 10\% FBS (Gibco, Life Technologies, Lofer, Austria), 100 units $/ \mathrm{ml}$ penicillin, and 100 units $/ \mathrm{ml}$ streptomycin (1705694, HyClone, USA) at $37^{\circ} \mathrm{C}$ in a $5 \%$ carbon dioxide humidified incubator. ISL were treated with two concentrations $(50 \mu \mathrm{M} / 100 \mu \mathrm{M})$ respectively for 5 hrs before inducing with $2 \mu \mathrm{g} / \mathrm{ml}$ LPS.

Immunohistochemistry 
Formalin-fixed renal tissues were paraffin-embedded and cut into $2 \mu \mathrm{m}$ thick. The sections were deparaffinized and rehydrated as routine protocols. Tissues were subjected to immunohistochemical staining for GPX4 (1:200, abcam, ab955) and xCT (1 $\mu \mathrm{g} / \mathrm{ml}$, ab64693, abcam). The process was conducted in strict accordance with the kit protocol. The images were taken by an Olympus Coolpix-micro digital camera from Japan.

\section{Electron Microscope (EM)}

For transmission electron microscopy, the kidney cortex was blocked in Fixative (G1102, Servicebio, China) for TEM at $4^{\circ} \mathrm{C}$ at least 2-4 h, and then, washed in $0.1 \mathrm{M}$ PBS for three times, 15 min each. Kidney tissues were post-fixed with 1\% OsO4 in 0.1M PBS ( $\mathrm{pH} 7.4)$ for $2 \mathrm{~h}$ at room temperature, dehydrated in graded ethanol and embedded by baking in $60^{\circ} \mathrm{C}$ oven for $48 \mathrm{~h}$. Ultrathin sections ( $60 \sim 80 \mathrm{~nm}$ thick) were stained with uranyl acetate and lead citrate and observed with TEM (HT7700, HITACHI, Japan).

\section{HE And PAS Staining}

Formalin-fixed renal tissues were paraffin-embedded and cut into $2 \mu \mathrm{m}$ thick. The sections were stained with Hematoxylin and Eosin (H/E) and PAS. The images were taken by an Olympus Coolpix-micro digital camera from Japan under $\times 400$ original magnification.

Renal Function Assessment

Blood samples were separated by $3,000 \mathrm{rpm}$ for $10 \mathrm{~min}$ at $4{ }^{\circ} \mathrm{C}$. Thereafter, serum was stored at $-80^{\circ} \mathrm{C}$. Serum blood urea nitrogen (BUN) and creatinine (CREA) were detected by BUN Assay Kit (C013-2) and Creatinine Assay Kit (C011-2) from Jiancheng, Nanjing, China.

Western Blot

The proteins of kidney tissues and HK2 cells was lysed by RIPA lysis buffer (\#P0013B, Beyotime, Shanghai, China) with 1\% PMSF (P0100, Solarbio, China). Resulted proteins were subjected to 10\% SDSPAGE. And then transferred to PVDF membranes (R7CA6580A, Thermo Fisher Scientifc, USA). The membranes were probed overnight with Beclin-1 (1:1000, 3495S, Cell Signaling Technology, USA), ATG7(1:1000, 8558S, Cell Signaling Technology, USA), GPX4 (1:1000, ab125066, abcam), NCOA4 (1:1000, abs-134557, Absin, China). The membranes were respectively incubated with HRP labeled goat anti-rabbit IgG $(1: 5000,511203$, Zen BioScience) and, HRP labeled goat anti-mouse IgG $(1: 5000,511103$, Zen BioScience). The bands were detected with enhanced chemiluminescence (1701102, Millipore, USA). The protein levels were normalized by $\beta$-actin (1:5000, HRP-60008, Proteintech).

Assessment of Nitric Oxide (NO), Malondialdehyde (MDA) and Superoxide Dismutase (SOD)

The level of NO, MDA and SOD were detected by NO assay kit (A012, Jiancheng, Nanjing, China), MDA assay kit (A003-1-2, Jiancheng, Nanjing, China) and SOD assay kit (A001-3, Jiancheng, Nanjing, China) following the manufacturer's instruction. 
Statistics Analysis

The results were expressed as means \pm standard deviation (SD). Statistical analysis was performed using Graph Pad Prism 5 software by one-way ANOVA with post hoc test. Newman-Keuls multiple comparison test was used to compare differences. Differences between groups were indicated statistically significant at $\mathrm{P}<0.05$.

\section{Results}

ISL prevents renal tubular epithelial injury in LPS-induced AKI

To validate ISL can ameliorated renal dysfunction in LPS-induced AKI, animal model was generated in mice by the injection of LPS and confirmed by HE staining (Fig. 1A). After LPS injection, renal tubules had moderate/severe degree injury, compared with control group. ISL treatment revealed significant protective effect which could ameliorate the renal tubules injury into mild degree in kidney tissue.

Ferroptosis involved in the protective effect of ISL in LPS-induced mouse model.

GPX4, the most important marker of ferroptosis, decreased in LPS group, especially in kidney tubules. $\mathrm{XCT}$ involved in an important pathway of ferroptosis. We found the expression of $\mathrm{XCT}$ expressed in glomeruli of kidney mainly and showed decreased in LPS group. Whereas ISL could increase the expression of GPX4 and xCT which protect tissues from ferroptosis. Electron microscope showed the mitochondrial changes in LPS group, including smaller volume with increased membrane density, a hallmark of ferroptosis, in contrast to control (Fig. 1A). Ferrostation-1(Fer) is an effective inhibitor of Ferroptosis. In vivo, we established LPS-induced mouse model, and we found both ISL and Fer could reduce the level of CREA and BUN (Fig. 1B). It revealed ISL and Fer protected renal function from LPSinduced AKI. Above these results, we hypothesized that ferroptosis might involve in LPS-induced AKI and the protective effect of ISL.

ISL inhibited the expression of lipid peroxidation both in vivo and vitro

Lipid peroxidation is a promoter of ferroptosis. Thus, we identified the level of MDA, SOD and nitrite in mice model. LPS injection could increase MDA, SOD and nitrite production. ISL treatment decreased the expression of MDA and nitrite remarkably. In the meantime, ISL could decrease SOD lightly to be protective (Fig. 2). To observe these findings in vitro, we established LPS-induced cell model in HK2 cell line. There are two concentrations of ISL in cells experiments. We divided cells into six groups, including control, ISL $50(50 \mu \mathrm{M})$, ISL $100(100 \mu \mathrm{M})$, LPS $(2 \mu \mathrm{g} / \mathrm{ml})$, LPS plus ISL $50(50 \mu \mathrm{M})$, and LPS plus ISL 100 $(100 \mu \mathrm{M})$. LPS could increase MDA and nitrite expression. With ISL treatment, MDA production decreased sharply. Additionally, more SOD production to maintain homeostasis after ISL treatment. As for nitrite, Fig. 2 showed two concentration of ISL could decrease NO. But there is a dramatical decrease after $100 \mu \mathrm{M}$ ISL instead of $50 \mu \mathrm{M}$, in contrast to LPS.

ISL inhibited LPS-induced autophagy, ferritinophagy and ferroptosis both in vivo and vitro 
Autophagy has been implicated in the pathogenesis of various diseases and biological processes. However, whether autophagy is injurious or protective for AKI has not clearly clarified. To validate the function in our model, we detected the most important markers which contribute to autophagy, including ATG7 and Beclin-1. The results showed ATG-7 and Beclin-1 were all elevated after LPS treatment, whereas ISL could inhibit these makers of autophagy both in vivo and vitro (Fig. 3). Ferritinophagy is newly discovered selective autophagy. Overactivated ferritinophagy could induce cellular overload iron and then might participate in ferroptosis, so we examined the expression of NCOA4 which recognized as a specific carrier for ferritinophagy. We found LPS could trigger NCOA4 expression. After ISL treatment, NCOA4 expressed less in contrast to LPS group both in vivo and vitro. More precisely, $100 \mu \mathrm{M}$ ISL in LPS-induced cells showed a significant decreased accumulation of NCOA4 (Fig. 4). GPX4 is identified as the most highly ranked candidate of biomarker in ferroptosis ${ }^{18}$. To examine the involvement of ferroptosis, we used western blot to show that GPX4 decreased in LPS-induced AKI which followed as autophagy biomarker. ISL can contribute to the accumulation of GPX4 both in normal and septic model. We highly considered that ferroptosis in LPS-induced AKI is an autophagy-related process.

HMGB1 might be a potential regulator of ISL in autophagy-related ferroptosis.

HMGB1 is required for LPS-induced autophagy in renal tubular. In the model of septic AKI, we found GPX4 decreased markedly, indicating that ferroptosis participated in this disease process. Interestingly, HMGB1 expression had the same trend as GPX4 (Fig. 4). The results suggested that HMGB1 involved in autophagy-related ferroptosis. But HMGB1 expression was blocked after ISL treated, which revealed ISL promoted abundant GPX4 to maintain the cellular homeostasis by suppressing HMGB1.

\section{Discussion}

AKI is a multifactorial renal disease characterized by a rapid decrease of renal function, resulting in the accumulation of toxins and failure of other organs ${ }^{19}$. Clinically, sepsis and ischemia-reperfusion injury (IRI) are the main causes of AKI ${ }^{19}$. The effective intervention in the early stage needs to be found. ISL, isolated form the root of licorice, has studied protective effect in multiple disease. Researchers found biological activities such as anti-inflammatory, anti-oxidative, anti-nephritic and anti-cancer activities ${ }^{15}$.

In our study, we established LPS-induced AKI model both in vivo and in vitro. We used the renal tissue from mouse model to do the H\&E staining and found LPS injection leaded the renal tubules to serious damage. However, ISL treatment can attenuate the injury in pathology. In the previous study, we confirmed that ISL can decline the CREA and BUN to ameliorate renal function of LPS-induced $\mathrm{AKI}^{20}$. These results showed the preliminary protective effect of ISL.

But the mechanism needs to be clarified. Multiple mechanisms participate in LPS-induced AKI. As an acute inflammatory disease of renal tubular cell, necrosis and apoptosis are noticeable cell death pathway currently ${ }^{19}$. However, in 2012, Dixon S et al. first demonstrated ferroptosis which is an irondependent form of cell death ${ }^{21}$. We hypothesis ferroptosis as a cell death pathway, involving LPS- 
induced AKI. At first, we used IHC to examine the protein expression. GPX4, identified as the most highly ranked candidate of biomarker in ferroptosis ${ }^{18}$, decreased in LPS group, especially in kidney tubules. XCT is also involved in an important pathway of ferroptosis. The expression of XCT showed decreased in LPS group. Electron microscope is a gold standard for ferroptosis. The result showed ISL ameliorated the mitochondrial damage in LPS group. Lipid peroxidation accumulation promotes ferroptosis. Thus, we identified the level of MDA, SOD and nitrite both in vivo and in vitro. LPS injection increased MDA and nitrite production, meanwhile SOD decreased which identified as antioxidant defense. ISL treatment decreased MDA and nitrite remarkably and increased SOD lightly. Above these results, we considered that ferroptosis might involve in LPS-induced AKI and the potential mechanism of ISL.

Autophagy has been implicated in the pathogenesis of various diseases and biological processes. However, whether autophagy is injurious or protective for AKI has not clearly clarified. To validate the function in our model, we detected the most important markers which contribute to autophagy, including ATG7 and Beclin-1. We demonstrated autophagy exist in LPS-induced AKI, whereas ISL could inhibit autophagy both in vivo and vitro. R. Kang et.al considered Beclin-1 is a key regulator of autophagy, can promote ferroptosis through a Beclin1-SLC7A11 complex ${ }^{22}$. The study showed that ferroptosis can be inhibited when knockout ATG $7^{23,24}$. Torii $S$ et al. validated that autophagy is required for the induction of ferroptosis both in normal and cancer cells ${ }^{25}$. Above these studies, we inferred the ferroptosis in LPSinduced AKI is autophagy-mediated.

Ferritinophagy is newly discovered selective autophagy. Overactivated ferritinophagy could induce cellular overload iron and then might participate in ferroptosis. NCOA4 recognized as a specific carrier for ferritinophagy. We found LPS could trigger NCOA4 expression. After ISL treatment, NCOA4 expressed less than control group. Glutathione peroxidase (GPX4), an antioxidant enzyme and an inhibitor of selective ferroptosis, is highly expressed in NCOA4-null mice ${ }^{26}$. NCOA4-mediated ferritin degradation is involved in ferroptosis ${ }^{6}$. Torii $\mathrm{S}$ et al. also demonstrated autophagy can contribute to ferroptosis by degradation of ferritin ${ }^{25}$. Thus, we highly considered that autophagy activated ferroptosis via NCOA4-mediated ferritinophagy (Fig. 5). Further, HMGB1 is a DAMP released by ferroptotic cells in an autophagydependent manner ${ }^{23}$. In our study, HMGB1 expression had the same expression trend as GPX4, indicating HMGB1 expression might from ferroptosis.

\section{Conclusions}

In this study, we verified the anti-inflammatory effects of ISL in LPS-induced acute kidney injury both in vitro and vivo. Moreover, we found the mechanism was associated with ferroptosis and ferritinophagy. Besides, we considered the suppression of HMGB1 in renal tubular against ferritinophagy was implicated in AKI. Ferrostatin-1, an inhibitor of ferroptosis, showed its protective function in vivo. However, further studies are awaited to confirm this preliminary outcome.

\section{Abbreviations}


AKI acute kidney injury

CKD chronic kidney disease

ISL isoliquiritigenin

LPS lipopolysaccharide

NO nitric oxide

ROS reactive oxygen species

MDA malondialdehyde

SOD superoxide dismutase

ICU intensive care unit

$\mathrm{RN}$ regulated necrosis

Fer Ferrostatin-1

EM electron microscope

\section{Declarations}

Ethics approval and consent to participate

There is no human study in our work. The study of animals has been granted by ethics committee of Sichuan provincial people's hospital.

\section{Consent for publication}

No applicable.

\section{Availability of data and materials}

The datasets used and/or analysed during the current study are available from the corresponding author on reasonable request.

\section{Competing interests}

The authors declare that they have no competing interests.

\section{Funding}


This work was supported by National Natural Science Foundation of China (81770742, 81700607 and 81800613), Youth Science and Technology Creative Research Groups of Sichuan Province (2015TD0013), PHD Foundation of Sichuan Academy of Sciences \& Sichuan Provincial People's Hospital (2015BS05), China Postdoctoral Science Foundation Funded Project (2016M592672), Foundation of Health and family planning commission in Sichuan (16PJ424, 17PJ060 and 16PJ425). Central university fund from UESTC (ZYGX2016J176) and Technology Supportive Plan of Sichuan Province (2015SZ0245). Sichuan Science and Technology Innovation Miaozi Project (2016063).

Dr. Yi Li host or participate in these funding mentioned above. All the funding to some extent had contribution to reagents purchase, experimental devices and consumables, animal and cell line culture, et al.

\section{Author Contributions}

YT completed the main experiments, and was a major contributor in writing the manuscript. YW, CW, MY, SC assisted experiments, and the order of them were followed by their experimental workload. LL contributed to IHC. GL and LW made contribution to analyzing the result of experiment and provide instructions. $M X$ and $Y L$ designed this work. $Y L$ also provided funding to complete this study. All authors read and approved the final manuscript.

\section{Acknowledgements}

Authors thank Prof. Shaoping Deng and Prof. Zhenglin Yang in University of Electronic Science and Technology, Sichuan Academy of Medical Sciences \& Sichuan Provincial People's Hospital for generously providing research platforms and technical support.

\section{References}

1. Waikar SS, Liu KD, Chertow GM. Diagnosis, epidemiology and outcomes of acute kidney injury. Clin J Am Soc Nephrol. 2008;3(3):844-861.

2. Sancho-Martinez SM, Lopez-Novoa JM, Lopez-Hernandez FJ. Pathophysiological role of different tubular epithelial cell death modes in acute kidney injury. Clin Kidney J. 2015;8(5):548-559.

3. Martin-Sanchez D, Ruiz-Andres O, Poveda J, et al. Ferroptosis, but Not Necroptosis, Is Important in Nephrotoxic Folic Acid-Induced AKI. J Am Soc Nephrol. 2017;28(1):218-229.

4. Friedmann Angeli JP, Schneider M, Proneth B, et al. Inactivation of the ferroptosis regulator Gpx4 triggers acute renal failure in mice. Nat Cell Biol. 2014;16(12):1180-1191.

5. Xie Y, Hou W, Song X, et al. Ferroptosis: process and function. Cell Death Differ. 2016;23(3):369-379.

6. Hou W, Xie Y, Song X, et al. Autophagy promotes ferroptosis by degradation of ferritin. Autophagy. 2016;12(8):1425-1428.

7. Mancias JD, Wang X, Gygi SP, Harper JW, Kimmelman AC. Quantitative proteomics identifies NCOA4 as the cargo receptor mediating ferritinophagy. Nature. 2014;509(7498):105-109. 
8. Tang M, Chen Z, Wu D, Chen L. Ferritinophagy/ferroptosis: Iron-related newcomers in human diseases. J Cell Physiol. 2018;233(12):9179-9190.

9. Lu B, Wang H, Andersson U, Tracey KJ. Regulation of HMGB1 release by inflammasomes. Protein Cell. 2013;4(3):163-167.

10. Wei S, Gao Y, Dai X, et al. SIRT1-mediated HMGB1 deacetylation suppresses sepsis-associated acute kidney injury. American journal of physiology. Renal physiology. 2019;316(1):F20-f31.

11. Song JX, Lu JH, Liu LF, et al. HMGB1 is involved in autophagy inhibition caused by SNCA/alphasynuclein overexpression: a process modulated by the natural autophagy inducer corynoxine $\mathrm{B}$. Autophagy. 2014;10(1):144-154.

12. Sun X, Tang D. HMGB1-dependent and -independent autophagy. Autophagy. 2014;10(10):1873-1876.

13. Xu T, Jiang L, Wang Z. The progression of HMGB1-induced autophagy in cancer biology. Onco Targets Ther. 2019;12:365-377.

14. Peng F, Du Q, Peng C, et al. A Review: The Pharmacology of Isoliquiritigenin. Phytother Res. 2015;29(7):969-977.

15. Hattori T, Sadakane C, Koseki J, Kase Y, Takeda S. Saireito probably prevents mesangial cell proliferation in HIGA mice via PDGF-BB tyrosine kinase inhibition. Clinical and experimental nephrology. 2007;11(4):275-282.

16. Lee SH, Kim JY, Seo GS, Kim YC, Sohn DH. Isoliquiritigenin, from Dalbergia odorifera, up-regulates anti-inflammatory heme oxygenase-1 expression in RAW264.7 macrophages. Inflammation research : official journal of the European Histamine Research Society ... [et al.]. 2009;58(5):257-262.

17. Yuan $X$, Zhang $B$, Chen $N$, et al. Isoliquiritigenin treatment induces apoptosis by increasing intracellular ROS levels in HeLa cells. Journal of Asian natural products research. 2012;14(8):789798.

18. Yang WS, SriRamaratnam R, Welsch ME, et al. Regulation of ferroptotic cancer cell death by GPX4. Cell. 2014;156(1-2):317-331.

19. Linkermann A, Chen G, Dong G, Kunzendorf U, Krautwald S, Dong Z. Regulated cell death in AKI. J Am Soc Nephrol. 2014;25(12):2689-2701.

20. Tang $Y$, Wang $C$, Wang $Y$, et al. Isoliquiritigenin attenuates LPS-induced AKI by suppression of inflammation involving NF-KB pathway. Am J Trans/ Res. 2018 15;10(12):4141-4151.

21. Dixon SJ, Lemberg KM, Lamprecht MR, et al. Ferroptosis: an iron-dependent form of nonapoptotic cell death. Cell. 2012;149(5):1060-1072.

22. Kang R, Zhu S, Zeh HJ, Klionsky DJ, Tang D. BECN1 is a new driver of ferroptosis. Autophagy. 2018;14(12):2173-2175.

23. Wen Q, Liu J, Kang R, Zhou B, Tang D. The release and activity of HMGB1 in ferroptosis. Biochem Biophys Res Commun. 2019;510(2):278-283.

24. Du J, Wang T, Li Y, et al. DHA inhibits proliferation and induces ferroptosis of leukemia cells through autophagy dependent degradation of ferritin. Free Radic Biol Med. 2019;131:356-369. 
25. Torii S, Shintoku R, Kubota C, et al. An essential role for functional lysosomes in ferroptosis of cancer cells. Biochem J. 2016;15;473(6):769-77

26. Bellelli R, Federico G, Matte A, et al. NCOA4 Deficiency Impairs Systemic Iron Homeostasis. Cell Rep. 2016;14(3):411-421.

\section{Figures}

A

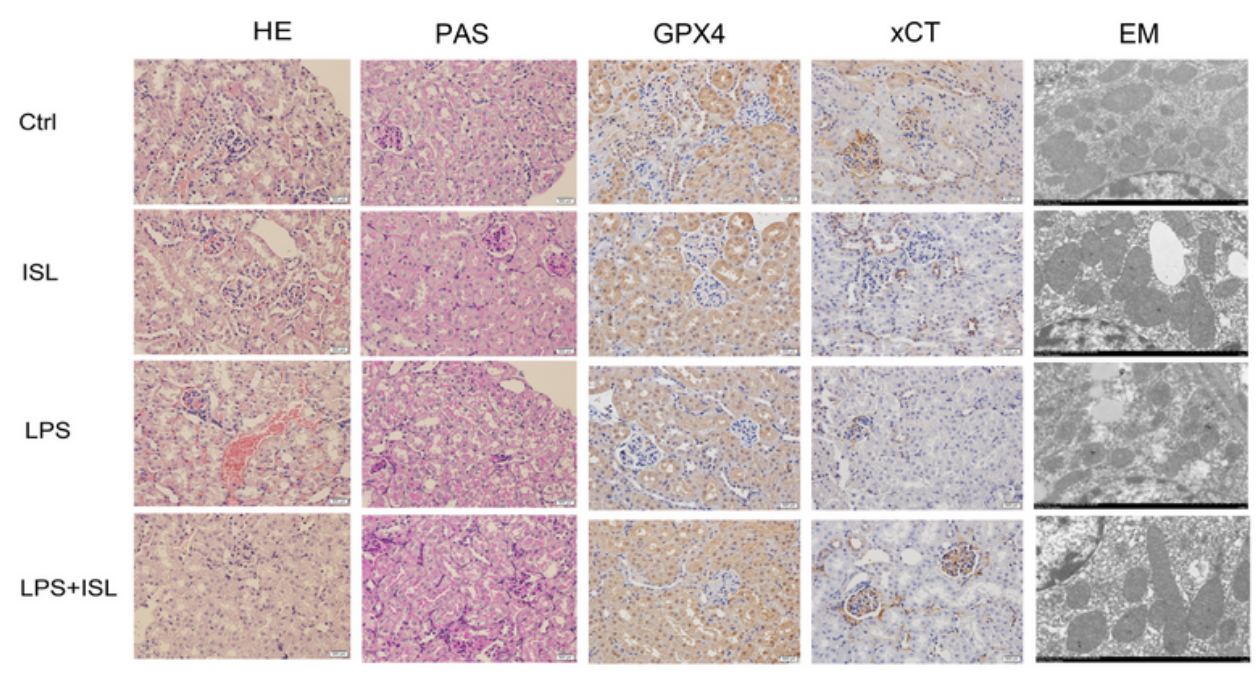

B
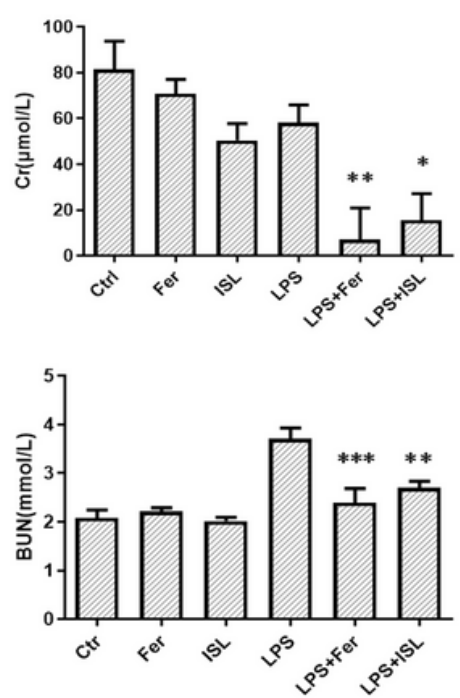

Figure 1

Ferroptosis involved in the protective effect of ISL in LPS-induced mouse model. A. HE staining and PAS staining showed the injury of renal tubular. ISL could reduce renal injury and thereby protect renal tubular from LPS-induced AKI. Immunohistochemistry showed the expression of GPX4 and XCT reduced in LPSinduced AKI, but elevated with ISL treatment. Mitochondrial damage mainly observed by EM. The structural alterations of mitochondria including abnormal mitochondria membrane swelling and disappeared mitochondrial crista found in LPS group. ISL had effective protection of mitochondria. B. Both ISL and Ferrostatin-1(Fer) reduced the level of CREA and BUN with LPS injection. * means comparation with LPS group and $P<0.05$. ** means comparation with LPS group and $P<0.01$. *** means comparation with LPS group and $\mathrm{P}<0.001$. 
A

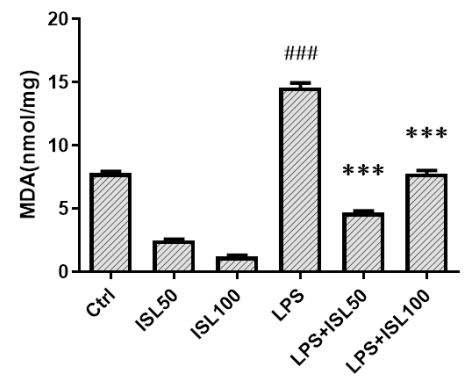

D

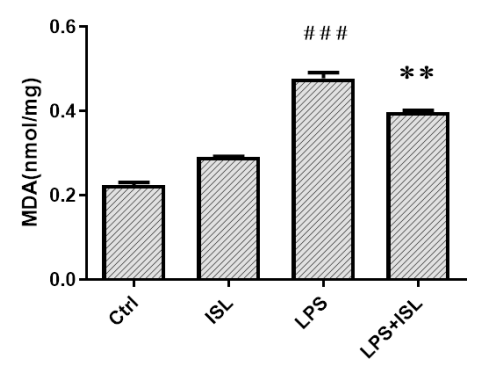

$B$

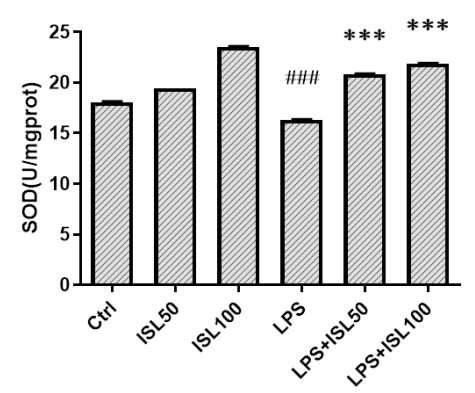

$E$

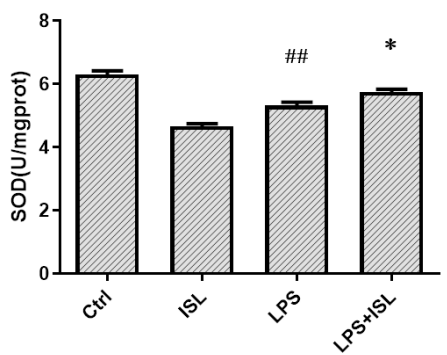

C

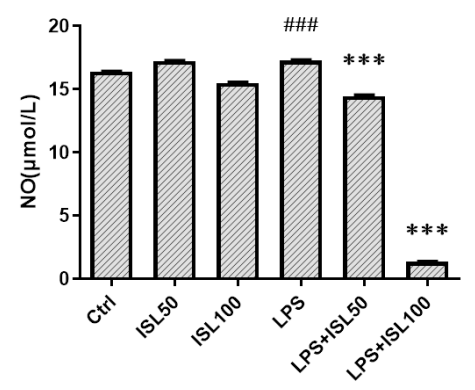

$\mathrm{F}$

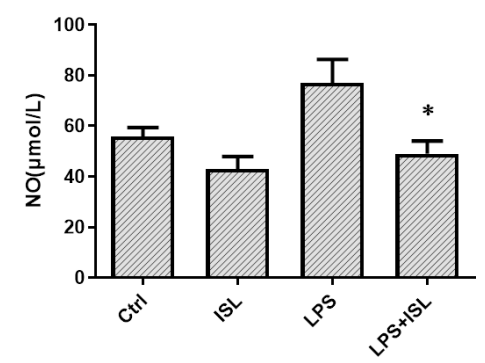

Figure 2

ISL inhibited the expression of lipid peroxidation in vivo and vitro. LPS-induced AKI presented as an increased level of lipid peroxidation (MDA and NO), but reversed trend in SOD. ISL inhibited the elevation of MDA and NO, and promoted the expression of SOD. A, B and C were tested in vitro and D, E and F were in vivo. * means comparation with LPS group and $\mathrm{P}<0.05$. ** means comparation with LPS group and $\mathrm{P}$ $<0.01$. *** means comparation with LPS group and $P<0.001$. \# means comparation with control group and $\mathrm{P}<0.05$. \#\# means comparation with control group and $\mathrm{P}<0.01$.\#\#\# means comparation with control group and $\mathrm{P}<0.001$. 
A

$B$

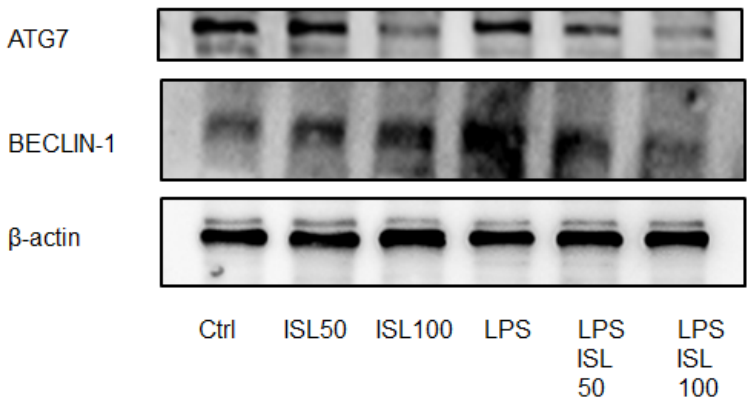

$75 \mathrm{kD}$

$52 \mathrm{kD}$

$46 \mathrm{kD}$

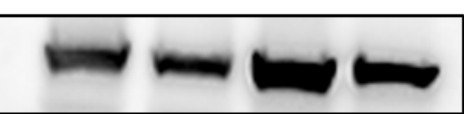

$75 \mathrm{kD}$

ATG7

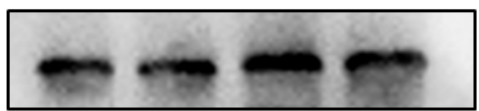

$52 \mathrm{kD}$

BECLIN-1

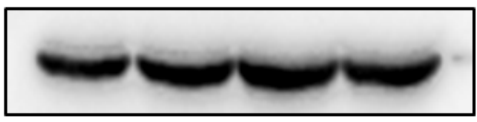

Ctrl

\section{Figure 3}

ISL inhibited LPS-induced autophagy both in vivo and vitro. The marker of autophagy including ATG-7 and Beclin-1 were detected by western blot. ISL attenuated LPS-induced autophagy.

A

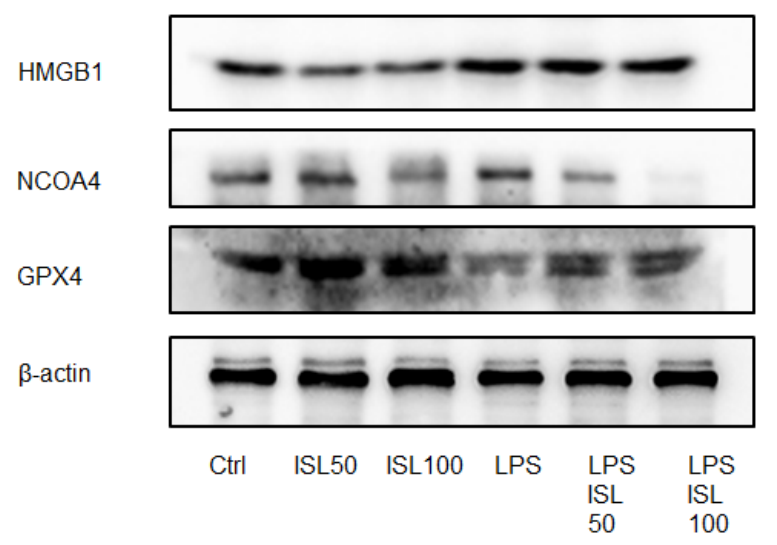

B

$30 \mathrm{kD}$

HMGB1

70kD NCOA4

$17 \mathrm{kD} \quad \mathrm{GPX} 4$

$46 \mathrm{kD}$

$\beta$-actin

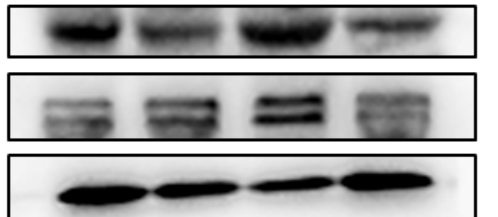

$30 \mathrm{kD}$

$70 \mathrm{kD}$

$17 \mathrm{kD}$

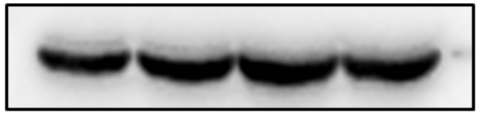

Ctrl ISL LPS LPS

Figure 4 
ISL attenuates LPS-induced ferritinophagy involving HMGB1. LPS activated NCOA4 but suppressed HMGB1 and GPX4. With ISL treatment, the expression of NCOA4 was reduced and HMGB1 and GPX4 were increased.

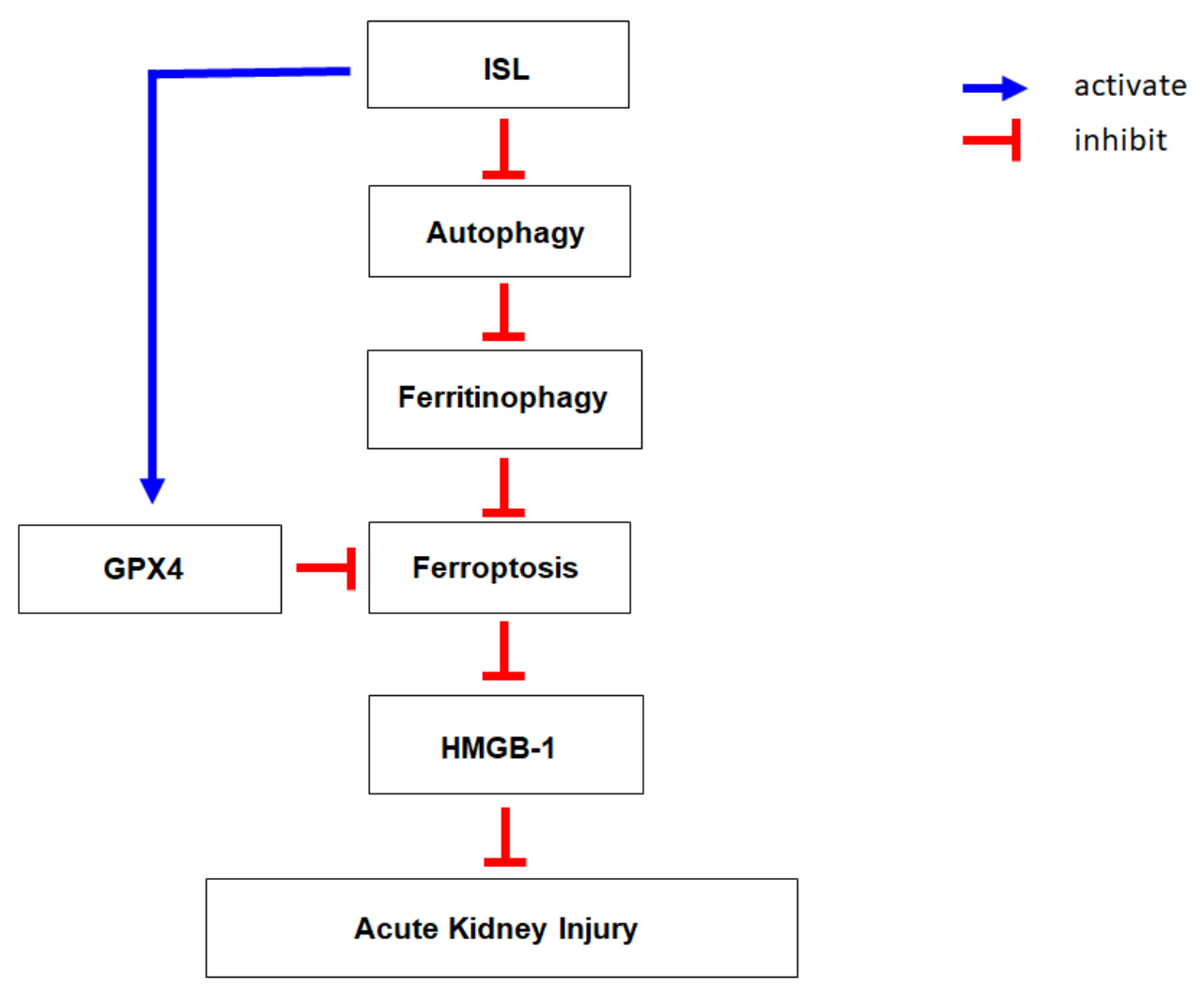

\section{Figure 5}

The mechanism diagram of ISL. We found ISL suppressed autophagy-mediated ferritinophagy resulting in inhibition of ferroptosis and the pathway involved HMGB1 which might be a potential regulator.

\section{Supplementary Files}

This is a list of supplementary files associated with this preprint. Click to download.

- NC3RsARRIVEGuidelinesChecklistfillable.pdf 